\title{
Users' Evaluation of Traffic Congestion in LTE Networks using Deep Learning Techniques
}

\author{
Bamidele Moses Kuboye \\ Department of Information \\ Technology, \\ Federal University of Technology, \\ Akure, \\ P. M. B. 704, Akure, Ondo State, \\ Nigeria
}

\author{
Tosin Opeyemi Aratunde \\ Department of Computer Science, \\ Federal University of \\ Technology,Akure, \\ P. M. B. 704, Akure, Ondo State, \\ Nigeria
}

\author{
Gbadamosi Ayomide A. \\ Department of Computer Science, \\ Federal University of \\ Technology,Akure, \\ P. M. B. 704, Akure, Ondo State, \\ Nigeria
}

\begin{abstract}
Deep learning is a division of machine learning built on a set of algorithms that attempt to model high-level abstractions in data by using prototypical architectures with complex structures. This work is based on using Deep learning to predict congestion on Long-Term Evolution (LTE). The work evaluates existence of traffic congestion in LTE networks using Convolutional Neural Networks (CNN) and Long Short-Term Memories (LSTMs) as Deep learning techniques. The accuracy from the results of both algorithms was compared to show the better algorithm on the prediction. The final accuracy of the deep learning model is given at $82 \%$ (0.82) which is the result of prediction with LSTM. Thus, LSTM proved to be more accurate in predicting the existence of congestion on the dataset. Prediction done with CNN and LSTM on the data collected showed that majority of LTE networks users suffer traffic congestion often.
\end{abstract}

\section{General Terms}

Deep learning, Congestion

\section{Keywords}

Long-Term Evolution (LTE), Convolutional Neural Networks (CNN), Long Short-Term Memories (LSTM), Algorithms, Traffic, subscribers

\section{INTRODUCTION}

Long Term Evolution (LTE) a broadband mobile communication technology that belongs to the Fourth generation $(4 \mathrm{G})$ of mobile communication was defined by 3rd Generation Partnership Project (3GPP). The main goals for LTE were increased peak data rates of downlink and uplink access, scalable bandwidth, enhanced spectral efficiency, increase in user capacity among other requirements. LTE met these requirements through the use of evolution of the Orthogonal Frequency Division Multiple Access (OFDMA), all IP flat architecture and evolved packet core (EPC). Evolved Universal terrestrial radio access networks (EUTRAN) and EPC formed Evolved Packet Systems (EPS) [1]. The universl acceptability of mobile broadband LTE led to growth in high bandwidth mobile applications like Multimedia Messaging Service (MMS), video sharing, Internet Protocol television (IPTV) and so on [2].

4G LTE has mobile data rate of up to $300 \mathrm{Mbps}$ theoretically exceeded the throughput of the Third Generation (3G) mobile technology. Due to the applications running on LTE, its subscriptions reached 1.2 billion in 2016 with about 150 million new subscriptions and an estimated subscription of up to 4.3 billion by the end of 2021 [3]. Nigeria has the largest mobile market in Africa with about 173 million subscribers and a penetration rate of $123 \%$ ) [4]. The Nigerian subsidiary of the MTN Group announced its financial report for the first quarter of 2020 and added 4.2million new subscribers in the first quarter. Its total number of subscribers became 68.5 million, the largest in the country. MTN's voice traffic and voice revenue were facing serious challenges [5]. Though voice traffic took a beat down, data traffic was rising fast which increased the level of congestion on the network. Traffic patterns changed following the presidential lockdown of Lagos, Abuja and Ogun States as part of initial responses to tackle the COVID-19 pandemic [6].

In response to the lockdown, data traffic spiked as subscribers began to adopt digital channels for most of their activities and routines including telecommuting, entertainment and social media engagements. People switched to WhatsApp, Telegram, Zoom and Google Meets for meetings, conferences and group calls. According to techcabal, in less than two months, Zoom grew from 10 million to 300 million users globally and telegram hit 400 million daily active users. Between January and March, the company's total number of active data subscribers jumped by 1.7 million to 26.8 million [5]. This trend increased the congestion rate on the network. MTN reported in April 2020 that its data traffic soared $135.6 \%$ year-on-year as more users worked the internet during the Covid-19 pandemic [7].

ML has been evolving as a discipline to the point that it currently allows wireless networks to learn and extract knowledge by interacting with data. [8]. Artificial Intelligence (AI), Machine Learning (ML), and Deep Learning (DL) are inter-related. The required information is learned directly from the data samples instead of complicated mathematical models in ML. ML automatically extracts complex features from data samples which are difficult for humans. Nevertheless, ML become difficult when the environments are heterogeneous, in other words, data collected from various sources have different formats, data demonstrate complex correlations, and have high dimensions. In such situations, significant human intervention is required to make accurate inferences and decisions based on the data. Deep learning (DL), which is a sub-class of ML, hierarchically extracts highlevel features and correlations from input data without human efforts through multiple layers of nonlinear processing units [9], [10]. The algorithms may be supervised or unsupervised. One of the promises of deep learning is replacing handcrafted features with efficient algorithms for unsupervised or semisupervised feature learning and hierarchical feature extraction [11]. Deep Neural Networks, Convolutional Deep Neural networks, and Recurrent Neural Networks are some of the DL architectures that have been applied to fields like computer 
vision, natural language processing, audio recognition and bioinformatics [12].

This research work focuses on evaluating users' perception on the traffic congestion in LTE networks using Deep learning techniques. This work evaluates perception of users on traffic congestion in LTE networks using Convolutional Neural Networks (CNN) and Recurrent Neural Networks (RNNs) or Long Short-Term Memories (LSTMs)) as Deep learning techniques and compare accuracy of the result. This work gives insight into how deep learning can efficiently be used to predict traffic congestion in LTE networks.

\section{REVIEW OF RELATED WORKS}

Ahmed et al [13] studied Deep Learning for Radio Resource Allocation in Multi-Cell Networks. This study was motivated by the increasing complexity and heterogeneity of emerging $5 \mathrm{G}$ and beyond 5G (B5G) wireless networks, which require a paradigm shift away from traditional resource allocation mechanisms. The aim of this research was to use deep supervised learning approach to solve the sub band and power allocation problem in multi-cell networks. The data was generated by genetic algorithm and the model was trained and tested with an accuracy of $86.3 \%$. Liang et al.[14] studied Deep Learning based Wireless Resource Allocation with Application to Vehicular Networks. The motivation behind this research is that wireless networks has become increasingly diverse and complex, e.g., in the high mobility vehicular networks, the current design methodologies face significant challenges and thus call for rethinking of the traditional design philosophy. The aim of this research was to leverage deep learning to solve difficult optimization problems for resource allocation and deep reinforcement learning for a direct answer to many resource allocation problems that cannot be handled or even modeled in the traditional optimization framework.

Hassan et al. [15]studied Machine Learning Approach to Achieving Energy Efficiency in Relay-Assisted LTE-A Downlink System. The research was motivated by the Energy Efficiency (EE) that has become a critical design metric for cellular systems. The goals of this research were to achieve $\mathrm{EE}$, a satisfactory balance between throughput and fairness. The work presents various resource block (RB) allocation schemes in relay-assisted Long Term Evolution-Advanced (LTE-A) networks. The dependency of RB and power allocation on relay deployment and users' association is first addressed through a k-means clustering approach. Furthermore, to reduce the computational cost of $\mathrm{RB}$ and power allocation, a two-step neural network $(\mathrm{NN})$ process was presented that uses unsupervised learning for RB allocation and supervised learning for power allocation in order to reduce computational cost of $\mathrm{RB}$ allocation. The research investigates the impact of using single and multiple L3 relays in terms of EE and throughput, the results does not give 100\% increase in $\mathrm{EE}$.

$\mathrm{Li}$ et al [16] presented the learning and prediction of application-level traffic Data in cellular networks. In this work, a significant amount of application-level traffic data from cellular network operators in china were collected, afterwards, a comprehensive study over the modeling and prediction framework of cellular network traffic was done. The results shows there exist universally some traffic statistical modeling characteristics like temporally-stable modeled property and spatial sparsity. Thereafter, a new traffic prediction framework was proposed to explore these aforementioned characteristics and then develop a dictionary learning-based alternating direction method to solve it. The simulation results demonstrated that the proposed framework could offer a unified solution for application-level traffic learning and prediction. Hancheng[17] studies Intelligent Resource Allocation Method for Wireless Communication Networks Based on Deep Learning Techniques. The concepts related to circuit switched computer network (CSCN) architecture are discussed and the throughput of small base stations (SBS) in CSCN architecture is analyzed. Thereafter, the long short-term memory network (LSTM) model is used to predict the mobile location of users, and the transmission conditions of users are scored based on the mobile location of users and the small base stations to which users are connected to have their desired cache states. The small base station selects several users with optimal transmission conditions based on the scores. Afterwards, the concept of game theory was used to model the problem of maximizing network throughput as a multi-intelligent non-cooperative game problem. A deep augmented learning-based wireless resource allocation algorithm was proposed to let the small base station learn autonomously and select channel resources based on the network environment so as to maximize the network throughput. The simulation results show that the algorithm proposed leads to a significant improvement in network throughput compared to the traditional random-access algorithm

Zhou et al [18] presented a paper on a deep-learning-based radio resource assignment technique for 5G Ultra Dense Networks. According to the work, it shows that 5G network operators need to carry out radio resource control in an efficient way rather than adopting the FDD/TDD scheme because the conventional uplink-downlink configuration change in the existing dynamic TDD method used for resource assignment is prone to repeated congestion. As a result, the issue was leverage using the deep LSTM learning technique to make localized prediction of the traffic load at the eNB. Simulation results showed that the proposed algorithm outperforms the conventional method in terms of packet loss rate, throughput, and MOS. Huang et al Studies the usage of Deep Learning Networks on Mobile Traffic Forecasting using the data traffic dataset based on call detail recording (CDR) the activities in the Milan city. Recurrent neural network (RNN), three-dimensional convolutional neural network (3D CNN), and combination of $\mathrm{CNN}$ and RNN (CNN-RNN) deep learning models were used. The work revealed that $\mathrm{CNN}$ and $\mathrm{RNN}$ can extract geographical and temporal traffic features respectively. Also, CNN-RNN proves to be a reliable model leading in all tasks with 70 to $80 \%$ forecasting accuracy when compared with other approaches, Zhao et al [19] studies Deep learning based mobile data offloading in mobile edge computing systems. The lack of resources in the licensed band because of the difficulties in have traffic data have prompted researches on mobile data offloading, as a result, WiFi AP is utilized to offload some devices from small base stations (SBS). Furthermore, a multi-Long Short Term Memory (LSTM) based deep learning model was used to predict the real-time traffic of SBS. Centered on the prediction results, a mobile data offloading strategy based on cross entropy (CE) method was proposed. The presented results provide strong proofs of the applicability of the prediction and offloading scheme proposed.

Bega et al [20] presented DeepCog; a data analytics tool for the cognitive management of resources in sliced 5G networks. DeepCog used a deep neural network structure to forecast rather than legacy mobile traffic prediction. Comparative 
evaluations with real-world measurement data, it allows for substantial reduction of about $50 \%$ or above of operating expenses with respect to resource allocation solutions. Guo et al [21] presented a paper on Proactive Dynamic Network Slicing with Deep Learning Based Short-Term Traffic Prediction for 5G Transport Network. The experimental results shows the mean utilization efficiency was improved from $46.33 \%$ to $71.53 \%$. Thantharate et al [22] presented a DeepSlice: A Deep Learning Approach towards an Efficient and Reliable Network Slicing in 5G Networks. The work used Deep Learning (DL) Neural Network to manage network load efficiency and network availability. The network Key Performance Indicators (KPIs) were used to train the model to analyze incoming traffic and predict the network slice for an unknown device type. The work claims that the intelligent resource allocation allows use of the available resources on existing network slic.es efficiently and offer load balancing.

\section{DATA COLLECTION AND ANALYSIS}

The data collected were from some universities in Nigeria using questionnaire on google forms so as to sample the satisfactions of subscribers on their mobile networks especially that the COVID-19 had made most university to adopt Hybrid classes. Most of the large classes are delivered online through Zoom, goolgle meet and the likes of such applications while the small classes are held physically but in observance of COVID-19 protocols. The responses from research questions that were sent to some students and 137 responded from Federal University of Technology, Akure (FUTA), ObafemiAwolowo University (OAU), University of Ibadan (UI), University of Lagos (UNILAG), Federal University Oye-Ekiti (FUOYE), and Federal University of Technology, Minna (FUTMINNA). The figure in 1 to 3 showed responses from different schools where data was collected, the service provider each respondent uses and their application areas of data usage. Figure 4 shows the percentage of number of the respondents who uses 4G SIM card and those who do not use 4G SIM card
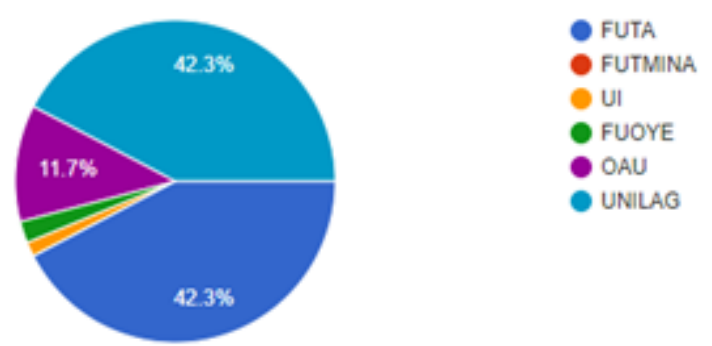

Figure 1: University that participated in the online Survey

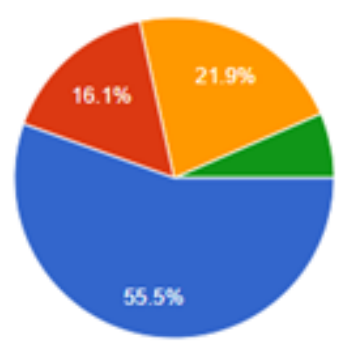

Figure 2: Service provider types

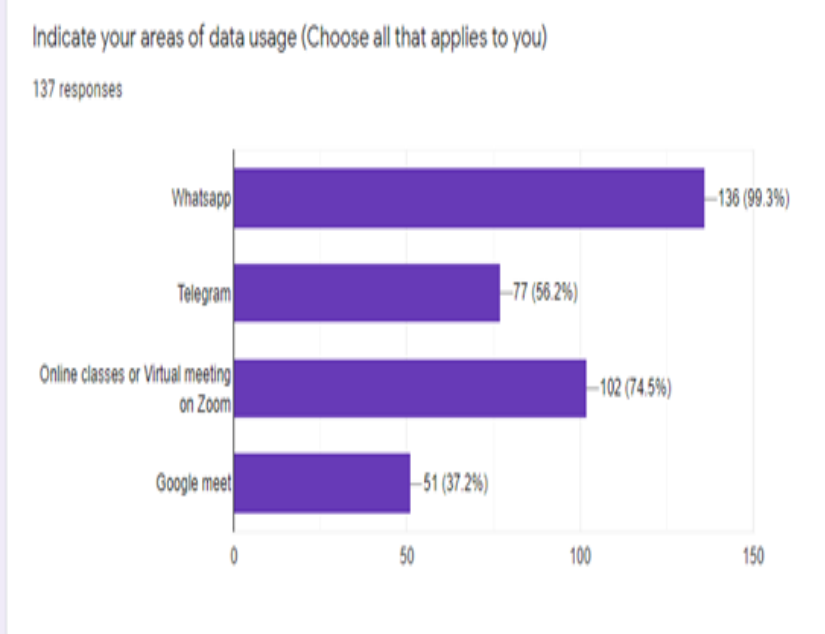

Figure 3: Type of applications

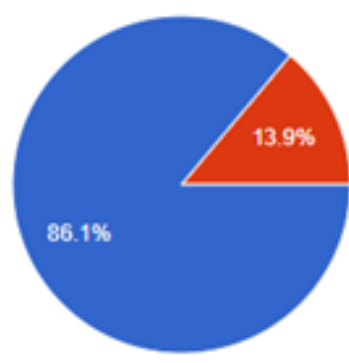

Figure 4: Percentage of Yes or No on 4G sim card enabled

\section{IMPLEMENTATION}

Jupiter notebook on anaconda was used as a framework to read the dataset using python code. The data were then encoded and trained using Convolutional Neural Networks (CNN) and Long Short Term-Memories (LSTMs)) as deep learning algorithms. Libraries in python such as pandas, numpy, sklearn and pickle were used in the process. The implementation involved Importation and viewing of data, and then data preprocessing process was done, that is, data encoding and checking for missing data. Thereafter, training using deep learning algorithms-CNN and LSTMs was done and Evaluation of the model on test data based on prediction with confusion matrix.

\subsection{Convolutional Neural Networks (CNN)}

In the process of training the data with $\mathrm{CNN}$, the data was partitioned into two parts; $70 \%$ for training and $30 \%$ for test. The training algorithm was used on the trained data of $70 \%$ and $30 \%$ was used for evaluation on the test data. Accuracy score on the trained data with CNN returns $76.8 \%(0.768)$. This means that $\mathrm{CNN}$ predict the existence of traffic congestion on training data with an accuracy of $76.8 \%$ (0.768). Figure 5 shows the graphical representation of the accuracy of $\mathrm{CNN}$ prediction on trained data. We ran training iterations (epochs) for $\mathrm{CNN}$ model, and accuracy increased during iteration. The plot shows the different stages of the accuracy in the iterations and the final accuracy is $76.8 \%$ 
(0.768).

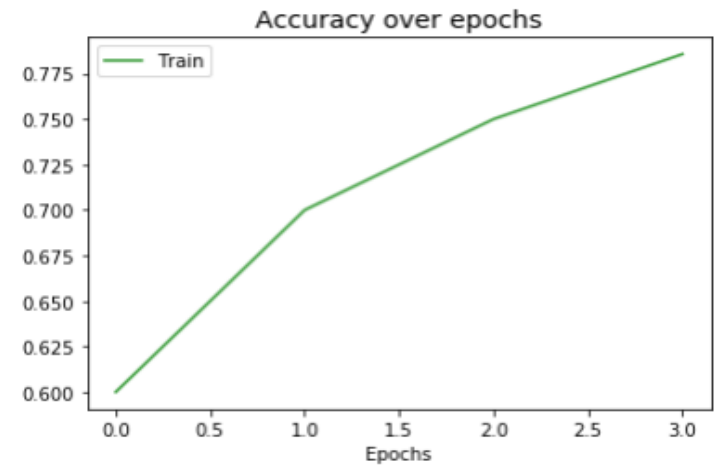

Figure 5: Graphical representation of CNN prediction on train data

print("accuracy score on test data: " + str(model.score(X_test, y_test)))

accuracy score on test data: 0.7857142857142857

\section{Figure 6: Training the test data with $\mathrm{CNN}$}

CNN was also used to train the test data and the accuracy score on test data returns $78.5 \%(0.785)$ as seen in figure 6 . This means that $\mathrm{CNN}$ predict the existence of traffic congestion on test data with an accuracy of $78.5 \%$ (0.785).

\subsection{Long Short Term Memories (LSTM)}

The graphical representation of the accuracy of LSTM prediction on trained data is shown Figure 7. We ran training iterations (epochs) for LSTM model, and accuracy increased during iteration. The plot shows the different stages of the accuracy in the iterations and the final accuracy is $82.2 \%$ $(0.8222)$. The prediction done using the test data and the result shows that majority of the subscribers experience traffic congestion.

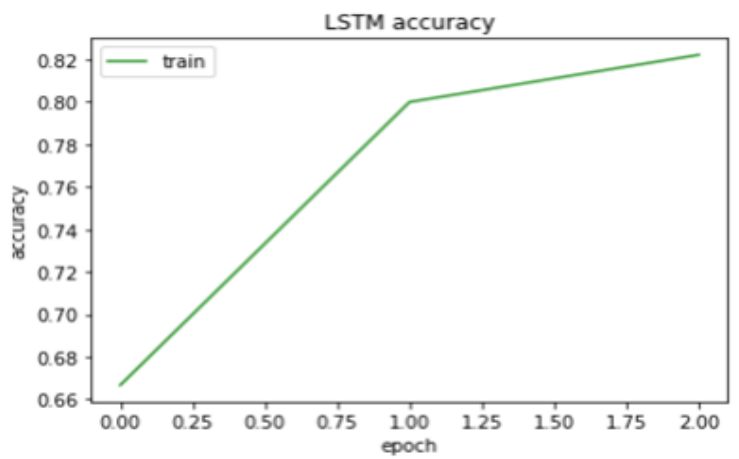

Figure 7: Graphical representation of LSTM prediction on trained data

\section{COMPARISON OF PREDICTION RESULT WITH CNN AND LSTM}

The graphical comparison of prediction result with CNN and LSTM is shown in Figure 8. The comparison showed that the accuracy of prediction with CNN is $76.8 \%$ (0.768) while the accuracy of prediction with LSTM is $82.2 \%$ (0.8222). Thus it is concluded that LSTM is more accurate in predicting the existence of congestion on the trained data.
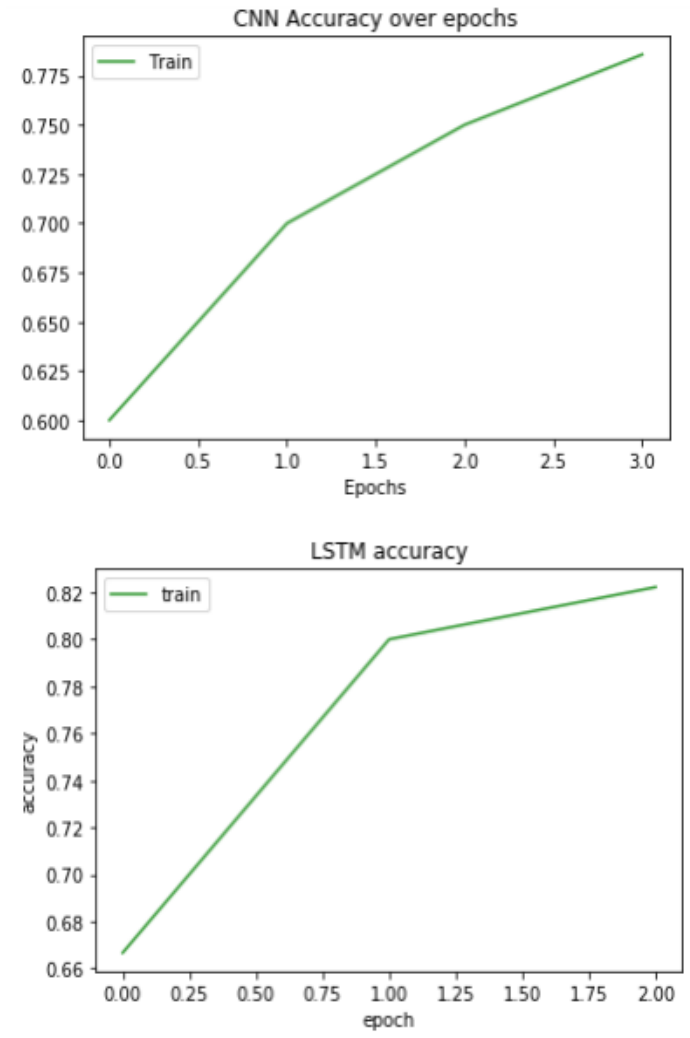

Figure 8: Graphical Comparison of Prediction Result with CNN and LSTM

\section{CONCLUSION}

LSTM proved to be more accurate in predicting the existence of congestion on the dataset given the final accuracy of the deep learning model is $82.2 \%(0.8222)$ as against $\mathrm{CNN}$ which gives $76.8 \%$ (0.768). Therefore, training on the dataset can be done directly with LSTM with expectation of a good accuracy on prediction. Prediction done with CNN and LSTM on the data collected showed that majority of LTE networks users suffers traffic congestion often. This work shows that deep learning algorithms can be used to predict the existence of congestion on the mobile network. Though this work shows that there is high level of traffic congestion among LTE users, but, it cannot precisely concluded since the dataset used were small. It will be very good if more data can be used so as to know the feelings of subscribers accurately. Nevertheless, it is recommended that telecommunication companies increase the bandwidth of their network so as to minimize congestion, thereby, increase the satisfaction of their subscribers. This will attract more subscribers to their network and invariably bring optimum gain. Furthermore, regular assessment should be carried out by operators so as to detect any shortcoming on the network and take necessary actions in order to satisfy the needs of subscribers. Also, it will be of great benefit if academic institutions can collaborate with network service providers for ease of access to dataset on network usage.

\section{REFERENCES}

[1] Kuboye B. M. (2021). Comparative analysis of scheduling algorithms performance in a Long Term Evolution Network, Journal of Computer Science Research 3(4). Doi: https://doi.org/10.30564/jcsr.v3i4.3555.

[2] Parikh Jolly (2016). Performance Analysis of LTE / TEAdvanced System, Doctor of Philosophy Ph.D.) Thesis 
submitted to the Department of Electronics and communication Engineering, Faculty of Engineering and Tech-nology, Mewar University, Chittorgarh

[3] Tchao E.T., J.D. Gadze and Jonathan ObengAgyapong (2018). Performance Evaluation of a Deployed 4G LTE Network. International Journal of Advanced Computer Science and Applications (IJACSA), Vol. 9, No. 3

[4] Telecommunication news. https://www.budde.com

[5] Financial reportvoicedatarevenue in Nigeria. www.techcabal.com/2020/04/30/mtn-q1-2020-inanacialreport-voice-data-revenue/.

[6] https://www.reuters.com/article/us-health-coronavirusnigeria-buhari.

[7] https://businesstech.co.za.

[8] Morocho-Cayamcela M. E, Haeyoung Lee and Wansu Lim (2016). Machine Learning For 5g/B5g Mobile And Wireless Comms.: Potential, Limitations, And Future Directions, vol 4, IEEEAccess

[9] Deng Li and Dong Yu (2014). Deep Learning. Methods and Applications LTE Overview - Tutorials point https://www.tutorialspoint.com/lte/lte_overview.htm

[10] Glauner, Patrick Oliver (2015). Deep Convolutional Neural Networks for Smile Recognition. Master of Science in Computing (Machine Learning) of Imperial College London

[11] Song and Lee (2013). Deep Learning and Neural Networks. Concepts, Methodologies, Tools, Google Books.

[12] Mathew Amitha, P.Amudha and S.Sivakumari (2020). Deep Learning Techniques. An Overview. https://www.researchgate.net/publication/341652370

[13] Ahmed K. I., H. Tabassum, and E. Hossain (2018). Deep Learning for Radio Resource Allocation in Multi-Cell Networks. arXiv:1808.00667v1 [cs.NI] 2 Aug 2018

[14] Liang Le, Hao Ye, Guanding Yu and Geoffrey Ye Li Fellow (2019). Deep Learning based Wireless Resource Allocation with Application to Vehicular Networks.

[15] Hassan Hammad, Irfan Ahmed, Rizwan Ahmad, HediKhammari, GhulamBhatti, Waqas Ahmed and Muhammad MahtabAlam (2019). A Machine Learning Approach to Achieving Energy Efficiency in Relay-Assisted LTE-A Downlink System. Sensors

[16] Li Rongpeng, Zhifeng Zhao, JianchaoZheng, Chengli Mei, YuemingCai, and Honggang Zhang (2017). The Learning and Prediction of Application-level Traffic Data in Cellular Networks.: arXiv

1606.04778v2 [cs.NI] 28 Mar 2017

[17] HuiHancheng, (2021). Intelligent Resource Allocation Method for Wireless Communication Networks Based on Deep Learning Techniques,Journal of Sensors, Volume 2021, Article ID 3965087, 12 pages, https://doi.org/10.1155/2021/3965087

[18] Zhou, Y., Fadlullah, Z. M., Mao, B., \& Kato, N. (2018). A Deep-Learning-Based Radio Resource Assignment Technique for $5 \mathrm{G}$ Ultra Dense Networks. IEEE Network, 32(6), 28-34. [8553651].

[19] Zhao Xianlong, Kexin Yang, Qimei Chen, Duo Peng, Hao Jiang, XianzeXu, XinzhuoShuang (2019). Deep learning based mobile data offloading in mobile edge computing systems, Future Generation Computer Systems, Volume 99, 346-355. https://doi.org/10.1016/j.future.2019.04.039,

[20] Bega, Dario, Marco Gramaglia, Marco Fiore, Albert Banchs, Xavier Costa-Perez. DeepCog: CognitiveNetwork Management in Sliced 5G Networks with Deep Learning. IEEE INFOCOM, Apr 2019, Paris, France. hal-01987878

[21] Guo, Qize, RentaoGu, Zihao Wang, Tianyi Zhao, YuefengJi, Jian Kong, RitiGour, Jason P. Jue (2019). Proactive Dynami c Network Slicing with Deep Learning Based Short-Term Traffic Prediction for 5G Transport Network.

[22] Thantharate, Anurag, Rahul Paropkari, Vijay Walunj, Cory Beard DeepSlice: A Deep Learning Approach towards an Efficient and Reliable Network Slicing in 5G Networks https://www.researchgate.net/publication/336179118, 978-1-7281-3885-5/19/\$31.00 